\title{
Face Recognition based on Sub-pattern Sparsity Preserving Projection
}

\author{
Qiwen Zhang ${ }^{1, a}$, Xinlei Zhuang, b \\ ${ }^{1}$ School of Computer and Communication, Lanzhou University of \\ Technology, Lanzhou, 730050, China \\ aemail: 823869941@qq.com, ${ }^{b}$ email:zxl198907@163.com
}

\begin{abstract}
In order to solve the problem of pseudo approach in SPP, an unsupervised algorithm named sub-pattern sparsity preserving projection(SpSPP) was proposed in this paper. In the proposed algorithm, face images are firstly divided into smaller sub-images, and sub-images from the same location are collected to compose the sub-pattern set. Then the conventional SPP is applied to each of sub-pattern sets to extract the local features. Finally, the sub-pattern features computed by SPP are concatenated to get the holistic features. Based on the fact that different regions of face images share a different similarity relationship and the discrimination information of sparse representation, SpSPP alleviated the effect of pseudo approach through image partition and feature concatenation. The effectiveness of the proposed method was verified on popular face databases (AR and Yale B).
\end{abstract}

Keywords: Face recognition; sparse representation; sparsity preserving projection; sub-pattern sparsity preserving projection

\section{Introduction}

Within the past decades, face recognition has become one of the most attractive fields of research and the most representative FR techniques are principal component analysis (PCA)[1] , locality preserving projection (LPP)[2] and sparsity preserving projection (SPP)[3]. In contrast to the above holistic methods which directly use whole face image for classification, some sub-pattern based techniques which extract facial feature from different levels of sub-patterns are proposed to overcome the ineffectiveness of holistic methods. In the sub-pattern methods, sub-pattern PCA (SpPCA)[4] was proposed by Chen. In their method, the whole images are firstly partitioned into smaller equal-sized sub-images in a non-overlapping way and the extraction of features is performed on each sub-pattern set composed by sub-images from the same location of original faces. By taking the different recognition contributions of different face parts into consideration, Tan extended the SpPCA to adaptively weighted sub-pattern PCA (Aw-SpPCA) [5]. In this approach, the weights of each sub-image set are determined by the similarities between the sub-pattern's probe 
set and gallery set. Introduced manifold learning theory into sub-pattern based algorithm, Ren presented sub-pattern locality preserving projection (Sp-LPP)[6]. In their method, they consider that not only whole faces, but sub-patterns of them also reside on a low-dimensional manifold subspace, and detect the low-dimensional manifold structure of different face regions.

In this paper, the theory of sub-pattern was introduced into SPP and a sub-pattern sparsity preserving projection (SpSPP) algorithm was presented for face recognition. The first step of SpSPP is to partition the original face images into several smaller equal-sized sub-images, and then all sub-patterns shared the same location are respectively collected to compose sub-pattern sets. In the second step, SPP is performed on each sub-pattern set to compute the transition matrix and extract sub-pattern features. Finally, the extracted features from the same face image are concatenated to a global feature, based on which the ultimate classification is processed. Experimental results on bench mark face databases showed that the proposed algorithm could effectively alleviate the influence of pseudo approach and achieve promising accuracy without any class information.

The rest of the paper is organized as follows: Section2 reviews SPP algorithm. The proposed SpSPP is introduced in Section 3. Experimental results and analyses are shown in Section 4. Finally, some conclusions are provided in Section5.

\section{Review of the Sparsity Preserving Projection}

Sparsity preserving projection considers the sparse reconstruction relationship of the data as a natural discrimination information and aims to preserve it in the embedded space. First of all, the sparse reconstruction weight vector $s_{i}$ for each $y$ is computed through the follow $l_{1}$ norm minimization problem in formula (1):

$$
\min _{s_{i}}\left\|s_{i}\right\|_{1} \quad \text { s.t. } \quad \begin{aligned}
& x_{i}=X s_{i} \\
& 1=I^{T} s_{i}
\end{aligned}
$$

where $s_{i}=\left[s_{i, 1}, \cdots, s_{i, i-1}, 0, s_{i, i+1}, \cdots, s_{i, n}\right]^{T}$ is the weight vector with the ith item equal to 0 . The component $s_{i j}, j \neq i$ in vector represents the contribution of sample $x_{j}$ to reconstruct $x_{i} . I \in R^{n}$ is a vector of all ones. Suppose that $\widetilde{S}_{i}$ is the optimal solution of formula (1), and then we get sparse reconstruction matrix $S=\left[\tilde{S}_{1}, \widetilde{S}_{2}, \cdots, \widetilde{S}_{n}\right]^{T}$.

Furthermore, under the situation of preserving the optimal weight vector $\widetilde{S}_{i}$, the SPP calculates the projection matrix by minimizing the objective function as follows, 


$$
\min _{T} \sum_{i}\left\|T^{T} x_{i}-T^{T} X \tilde{s}_{i}\right\|^{2}
$$

Let $e_{i}$ be a n-dimensional unit vector with the ith element 1,0 otherwise. With simple algebraic formulation, the objective function will be recast as the following optimization problem:

$$
X M X^{T} T=\lambda X X^{T} T
$$

where $M=S+S^{T}-S^{T} S$.

In an ideal situation, there are enough training samples of each class and all the testing sample $y$ of class $j$ will be faithfully represented by the over-complete dictionary composed by the samples of class $j$. Assume that $x_{i}^{j}$ is an arbitrary face sample of class $j$, then $x_{i}^{j}$ will be represented by the samples from class $j$ as shown in formula (4).

$$
x_{i}^{j}=0 \cdot x_{1}^{1}+\cdots+s_{i}^{1} \cdot x_{1}^{j}+\cdots s_{i}^{n_{j}} \cdot x_{n_{j}}^{j}+\cdots 0 \cdot x_{n}^{c}
$$

But in the scenario of face recognition, the insufficient of sample is a common problem. Thus the formula (4) will no longer hold, then SPP needs to take advantage of the similarity between heterogeneous samples to represent the sample collaboratively. But the chosen heterogeneous samples have a certain degree of uncertainty, which may project the samples apart in original space into a close position. In the other hand, SPP doesn't consider much about the heterogeneous samples close to the target sample which may lead these samples still close to target sample after projection. These two factors influence the recognition accuracy of SPP, and they were collectively referred to as pseudo approach in this paper.

\section{Sub-pattern Sparsity Preserving Projection}

Algorithm Procedure. In order to overcome the pseudo approach of SPP, a new sub-pattern based algorithm named sub-pattern sparse preserving projection (SpSPP) is proposed in this paper. Like other sub-pattern based techniques, SpSPP is also mainly consists of three steps. Firstly, face images are partitioned into equal-size sub-images in a non-overlapping way, and sub-images from the same location of face images are composed by the sub-pattern sets. Secondly, SPP is performed on each of sub-pattern sets to extract the local features, then the holistic feature is computed by concatenating the local features. Finally, classify unknown face images in the feature space.

4. Image partition

Assume $X=\left\{x_{1}, x_{2}, \cdots, x_{n}\right\}$ is a training set composed by $n$ face images, where $x_{i} \in R^{D}(i=1,2, \cdots, n), D$ present the dimensionality of 
observation space. For each sample of $X, X_{i}$ is partitioned into $N$ equal-sized sub-images $x_{i}^{l_{1}}, \cdots, x_{i}^{l_{N}}$ in a non-overlapping way. Then collect sub-images from the same location $x_{1}^{l_{i}}, \cdots, x_{n}^{l_{i}}$ into sub-pattern set $X^{l_{i}} i=1, \cdots, N$.

\section{Feature extraction}

After partition and collection we get $N$ sub-image sets, then local features are extracted by performing SPP on each sub-pattern set $X^{l_{i}}(i=1, \cdots, N)$. Suppose $x_{i}^{l_{i}}$ is a arbitrary sample of $X^{l_{i}}$, its sparse reconstruction vector $s_{i}^{l_{i}}$ is computed by using $X^{l_{i}}$ as the over-complete dictionary.

$$
\min _{s_{i}}\left\|S_{i}^{l_{i}}\right\|_{1} \quad \text { s.t. } \quad \begin{aligned}
& l_{i}^{l_{i}}=X^{l_{i}} s_{i}^{l_{i}} \\
& 1=I^{T} s_{i}^{l_{i}}
\end{aligned}
$$

Set $\widetilde{S}_{i}^{l_{i}}$ is the optimal solution of formula (5), we will get the sparse reconstruction matrix $S^{l_{i}}=\left[\widetilde{S}_{1}^{I_{i}}, \widetilde{S}_{2}^{I_{i}}, \cdots, \widetilde{S}_{n}^{I_{i}}\right\rfloor$.

Preserve the sparse reconstruction weight matrix $S^{l_{i}}$ and minimize the reconstruction error of every sample in embedded space, then we will get the objective function shown as follows:

$$
J(A)=\sum_{i}\left\|T^{l_{i}^{T}} x_{i}^{l_{i}}-T^{l_{i}^{T}} X^{l_{i}} \widetilde{s}_{i}^{l_{i}}\right\|^{2}
$$

With simple algebraic formulation, we get

$$
T^{l_{i}^{T}} X^{l_{i}}\left(I-S^{l_{i}}-S^{l_{i}^{T}}+S^{l_{i}} S^{l_{i}^{T}}\right) X^{l_{i}{ }^{T}} T^{l_{i}}
$$

Finally, the objective function can be recast as the following optimization problem:

$$
X^{l_{i}} M X^{l_{i}^{T}} T=\lambda X^{l_{i}} X^{l_{i}^{T}} T
$$

where $M=\left(I-S^{l_{i}}-S^{l_{i}^{T}}+S^{l_{i}} S^{l_{i} T}\right)$. Then the optimal transformation matrix $T^{l_{i}} \in R^{n \times d^{l_{i}}}$ is composed by the eigenvectors $w_{1}^{l_{i}}, w_{2}^{l_{i}}, \cdots, w_{d^{l_{i}}}^{l_{i}}$ corresponding to the largest $d^{l_{i}}$ eigenvalues of formula(8).

Finally, the global feature is obtained by combining all of the sub-pattern set features.

6. Classification unknown patterns

In the recognition phase, partition the unknown sample $U$ into 
$N$ sub-images and concatenate these images to a $D$ dimensionality vector at first. Then project the training samples into the $N d^{l_{1}}$ dimensionality feature space and classify it with a nearest-neighbor classifier.

Algorithm Analysis. In order to guarantee the sparse vector is computed under a over-complete system, the technique SPP is usually performed in a lower space projected by other dimensionality reduction method and which may lead the loss of effective information and affect the recognition rate. The proposed SpSPP first partitions face images into sub-images and then it performs SPP on sub-image set to alleviate this problem to a certain extent.

The conventional SPP has a certain robustness to illumination and expression changes, but in the situation of FR with insufficient data, this robustness is weakened profoundly. The proposed SpSPP execute SPP on sub-image sets, and the variations in illumination and expression will affect a few of them, hence we expect this method have better robustness than the conventional version.

Recently, almost every new proposed sub-pattern based method classify the query pattern in a ballot way. However, based on the analysis of SPP, we choose the more suitable feature combination to make the final decision. This suitable is mainly reflected in two aspects as follows:

1) In the practical application, voting method will face the situation that some candidates share the same votes. This problem can be solved through a weighted manner, but this needs extra class information. Another feasible method is to increase the voting number, which means the face images need to be divided into much smaller sub-images that may reduce the information carried by sub-images. In this case, the classification accuracy will be affected consequently. Moreover, the efficiency of algorithm will be affected at the same time.

2) Through the analysis of SPP algorithm mentioned before, the pseudo approach problem will affect the accuracy of algorithm and remedy this influence will improve the classification accuracy. Actually, different regions of face image have different similarity relationship (representation relation and instance relation). The different relation of representation makes the sparse vector of different sub-image computed on dictionary share different location of external nonzero entries. And the different relation of distance makes different sub-image share different neighborhood relationship, hence the distribution of samples affected by pseudo approach will disparate in different images. In addition, the discrimination of SPP guarantees the structure of homogeneous samples will be preserved in every sub-pattern sets. Therefore, the holistic feature concatenated by sub-pattern features will hold the relation of homogeneous datum and disperse the heterogeneous samples affected into several classes, which make the influence of pseudo approach alleviated and improve the effectiveness of classification. 


\section{Experimental results and analyses}

In this section, the performance of the proposed algorithm (SpSPP) is evaluated on two benchmark face databases (AR shown in Fig.1 and Yale B shown in Fig.2). There are four different methods used here for comparisons, they are SpPCA[4], SpLPP[6], SPP[3] and V-SpSPP (i.e. vote based sub-pattern sparse preserving projection). In order to guarantee the SPP is performed under a over-complete system, we project the original samples with PCA before the implement of SPP. The sub-pattern based algorithms are performed on different partitions (as shown in Fig. 3) respectively.

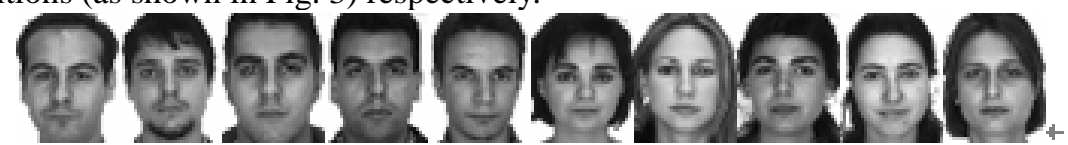

Fig.1 Face images in AR

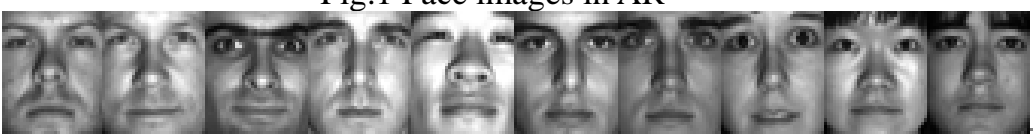

Fig.2 Face images in Yale B

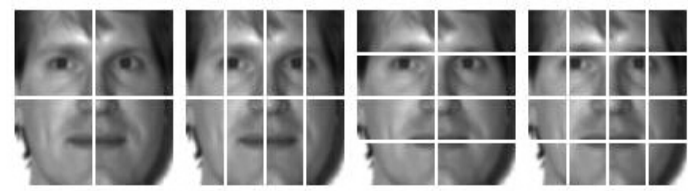

Fig.3 Partitions of face image

Fig. 4 shows the accuracy versus dimensions curve on AR database, and SpSPP achieves a recognition rate of 96.55, the best accuracies achieved by SpPCA, SpLPP and SPP are 71.55, 78.33 and 86.43 respectively. The V-SpSPP outperforms previous three techniques with a rate of 92.02. Fig. 5 shows the recognition rates on Yale B database, and the proposed SpSPP acquires a 97.40 recognition rate followed by 95.02 of V-SpSPP, 94.16 of SPP, 91.77 of SpLPP and 87.45 of SpPCA.

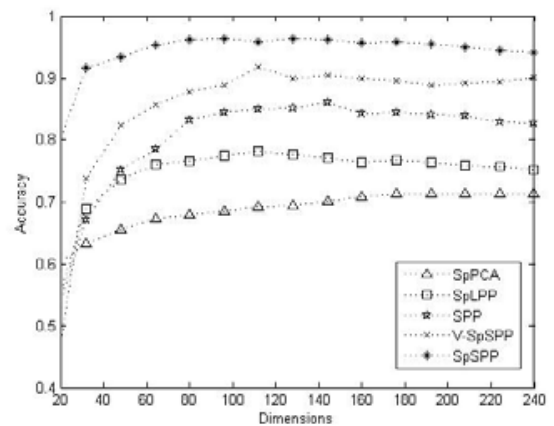

Fig.4 Accuracy on AR database 


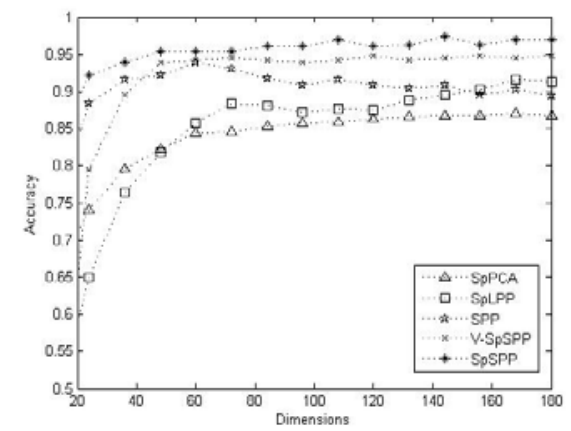

Fig.5 Accuracy on Yale B database

In order to testify the concatenation of features is more suitable for SPP based sub-pattern method, we record the classification accuracies of techniques V-SpSPP and SpSPP with the different image partition, and the consequences are showed in Table 1. From Table 1 we will see, SpSPP achieves an obvious precedency to V-SpSPP in classification accuracy with the $2 \times 2$ partition. With the increasing of sub-images, the recognition rate of V-SpSPP obtains a minor upgrade while the recognition rate of SpSPP declines significantly, but SpSPP can still classify the query samples in a similar accuracy with V-SpSPP.

Table 1 Recognition rates with different partition of images on the AR database

\begin{tabular}{ccccc}
\hline \multirow{2}{*}{ Method } & \multicolumn{4}{c}{ Different partition } \\
& $2 \times 2$ & $2 \times 4$ & $4 \times 2$ & $4 \times 4$ \\
\hline V-SpSPP & 91.90 & 90.71 & 91.67 & 92.02 \\
SpSPP & 96.55 & 95.00 & 91.79 & 91.71 \\
\hline
\end{tabular}

Based on the results of experiments on the AR database and Yale B database, we obtained the following conclusions:

1) The introduction of the sub-pattern based principle indeed improves the recognition rates of PCA and LPP in dealing with the face images affected by illumination and expression varied. However, the SpPCA and SpLPP are still unsupervised techniques, the embedded space detected by them is single manifold structure which difficult for classification.

2) The SPP algorithm based on sparse representation achieves a relative decent recognition rate on both databases, but the accuracy of SPP is not ideal enough due to the pseudo approach problem. The V-SpSPP and SpSPP obtain better classification accuracies without the use of label information, which is benefit from the alleviation of pseudo approach through multiple projection in sub-pattern based method.

3) With the increase of sub-patterns, the effective features carried by each sub-patterns declined significantly which weakened the discrimination of SPP. The augment of "vote number" fails to improve the accuracy obviously but make the extraction of features more time-consuming. However, the SpSPP used features concatenation as the identification 
strategy achieves a much more better accuracy in an efficient way.

\section{Conclusion}

In order to alleviate the pseudo approach in SPP, a sub-pattern sparse preserving projection (SpSPP) algorithm was proposed in this paper. Based on the fact that different regions of face images share different similarities, then the different sub-pattern sets have a different neighbor relation in embedded space. In additions, the discrimination of sparse representation will preserve the structure of homogeneous samples even with partial images. Combined these characters, SpSPP dispersed the influence of pseudo approach by extracting features on sub-pattern set respectively, and made the samples from the same class more tightly by concatenating these features. Experiment result on benchmark face image database AR and Yale B was verified the feature concatenation is a more suitable identification strategy for SpSPP, what's more, the proposed SpSPP was able to alleviate the pseudo approach problem and improve the accuracy of face recognition.

\section{Acknowledgement}

In this paper, the research was sponsored by the National Natural Science Foundation of China (No. 61263047) and Nature Science Foundation of Gansu Province (No. 2011GS04147).

\section{References}

[1] Turk M, Pentland A. Eigenfaces for recognition[J]. Journal of cognitive neuroscience, 1991, 3(1): 71-86.

[2] Xiaofei He, Shuicheng Yan, Yuxiao $\mathrm{Hu}$, et al. Face recognition using Laplacianfaces[J].IEEE Transactions on Pattern Analysis and Machine Intelligence, 2005, 27(3): 328-340.

[3] Lishan Qiao, Songcan Chen, Xiaoyang Tan. Sparsity preserving projections with applications to face recognition[J]. Pattern Recognition, 2010, 43(1): 331-341.

[4] Songcan Chen, Yulian Zhu. Sub-pattern based principal component analysis[J].Pattern Recognition, 2004, 37(1):1081-1083.

[5] Keren Tan, Songcan Chen. Adaptively weighted sub-pattern PCA for face recognition[J]. Neurocomputing, 2004, 64(3):505-511.

[6] Chengjuan Ren, Shufang Hu, Chengwen Liu. Sub-pattern locality preserving projection for face recognition[J].Journal of Chongqing University of technology(Natural Science), 2011,25(6):84-89. (In Chinese) 